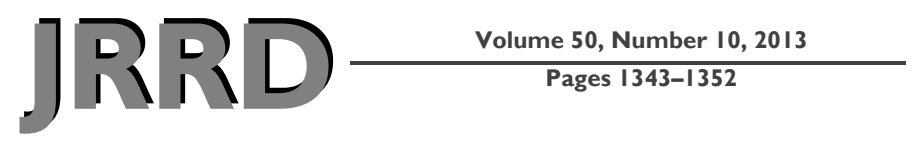

\title{
Evaluating Barten image metric for predicting character recognition in people with low vision
}

\author{
Kimberly A. Schoessow, OTD, OTR/L; ${ }^{1-2}$ Lisa M. Mauney, MS; ${ }^{1}$ Mark Uslan, MS; ${ }^{3}$ Ronald A. Schuchard, PhD ${ }^{1,4^{*}}$ \\ ${ }^{1}$ Rehabilitation Research and Development, Department of Veterans Affairs Palo Alto Health Care System, Palo Alto, \\ $C A ;{ }^{2}$ The Frank Stein and Paul S. May Center for Low Vision Rehabilitation at California Pacific Medical Center, San \\ Francisco CA; ${ }^{3}$ AFB Tech, American Foundation for the Blind, Huntington, WV; ${ }^{4}$ Department of Neurosurgery, Stanford \\ University School of Medicine, Stanford, CA
}

\begin{abstract}
Electronic devices with small visual displays (SVDs) are often inaccessible to the millions of Americans with vision loss. The Barten square root integral (SQRI) is an image quality metric that has been shown to predict whether people with normal vision can see images on a cathode ray tube monitor. The present proof-of-concept study begins to explore whether the same metric could predict the ability of users with low vision to see images on SVDs. In a sample population of 33 adults with low vision, the Barten SQRI was the best predictor of the ability to recognize low-contrast single digits on a screen $\left(r^{2}=0.80, p<0.01\right)$, followed by the PelliRobson Contrast Sensitivity Chart $\left(r^{2}=0.69, p<0.01\right)$. Visual acuity was not significantly predictive of the ability to read low-contrast characters on a display. Further work will explore whether the Barten SQRI remains predictive of the ability of people with low vision to use actual devices that have SVDs.
\end{abstract}

Key words: accessibility, contrast sensitivity, image metric, low vision, modulation transfer function, reading, resolution, small visual displays, universal design, vision rehabilitation.

\section{INTRODUCTION}

In today's high-tech environment, the use of electronic devices with small visual displays (SVDs) is an everyday occurrence. The potential use of devices with SVDs is present in all eight areas of occupation identified by the American Occupational Therapy Association [1]: personal activities of daily living (blood glucose monitors), instrumental activities of daily living (kitchen appliances), rest and sleep (alarm clocks), education (e-readers and tablet computers), work (fax and copy machines), play (video games), leisure (MP3 and DVD players), and social participation (cellular telephones). In developed countries, rarely does someone go through a day without interacting with these types of devices.

Use of these electronic devices with SVDs can be difficult to impossible, however, for the millions of Americans with low vision associated with eye diseases, neurological conditions, and aging. Some devices have been adapted to be easier to see or to be used with alternative senses. Smartphones and tablet computers usually have built-in magnification software, MP3 players often

Abbreviations: $\mathrm{CRT}=$ cathode ray tube, $\mathrm{CSF}=$ contrast sensitivity function, IRB = institutional review board, $\mathrm{LCD}=$ liquid crystal display, $\mathrm{MTF}=$ modulation transfer function, $\mathrm{PRL}=$ preferred retinal locus, SKILL = Smith-Kettlewell Institute Low Luminance, SLO = scanning laser ophthalmoscope, SQRI = square root integral, SVD = small visual display, ViSaGe = Visual Stimulus Generator.

*Address all correspondence to Ronald A. Schuchard, PhD; VA Palo Alto Health Care System (151A), 3801 Miranda Ave, Palo Alto, CA 94304; 650-493-5000, ext 64536; fax: 650852-3203. Email: rschuch@stanford.edu

http://dx.doi.org/10.1682/JRRD.2012.09.0164 
have speech output, and "talking" glucometers and blood pressure monitors exist. Even these adapted devices have functional limitations [2], however, and the majority of electronics with SVDs have no accessibility options. This presents a barrier to participation in meaningful roles and activities for people with low vision. It also places a financial burden on the individual, the family, and society when people can no longer independently manage their medications, finances, or home management tasks. This increased need for assistance can lead to excess disability, overprotection, caregiver burden, and negative interpersonal relationships within social networks [3-7].

It is crucial that people with visual impairments be given improved access to SVDs on electronic devices. There are two levels at which this can be accomplished. The first method requires manufacturers to create devices using principles of universal design with displays having optimum contrast, reflection, and font size. Rather than taking these ideal visual characteristics into account, manufacturers currently base their practices on inexpensive and readily available liquid crystal display (LCD) technology [8-10]. Providing specific guidelines about which characteristics enhance usability of displays could assist manufacturers in producing devices that are accessible to the millions of people with low vision. The second method for increased access is improvements in vision rehabilitation treatments and technology. A more thorough understanding of how individuals with low vision interact with and use SVD devices could inform rehabilitation professionals' decision-making processes as they work with clients to utilize ideal magnifiers, lighting, visual skills, and adaptive techniques.

One potential tool to assist manufacturers in designing more accessible SVDs and to help rehabilitation professionals choose techniques and visual aids that will allow clients to access SVDs is the Barten square root integral (SQRI) image quality metric. The Barten SQRI is an image metric that was originally developed to measure whether people with normal vision could see images on cathode ray tube (CRT) televisions and computer monitors. One component of the Barten SQRI, the modulation transfer function (MTF), describes how an imageforming system (display) filters the modulation (contrast) as a function of spatial frequency [11]. In other words, the MTF is a measure of how an electronic device transmits the contrast levels of the images it produces on a display. However, it is difficult to measure the perceived quality of an image because it depends on both the physi- cal parameters (e.g., MTF) of the system as well as how the visual system (eye and brain) perceives the image. An image quality metric - a mathematical expression that incorporates the physical parameters of the image produced by the system with the characteristics of the human observer - can be used to measure the perceived quality of an image. The Barten SQRI has been shown to adequately describe the performance of the combined human and image forming system. The Barten SQRI image metric is given by (Equation (1)) -

$$
S Q R I=\frac{1}{\ln 2} \int_{u_{1}}^{u_{2}} \sqrt{\frac{M(u)}{m_{t}(u)}} \frac{d u}{u},
$$

where $d u / u=$ differential of the logarithm of the spatial frequency $d(\log u), u_{1}$ and $u_{2}=$ the lower and upper observable spatial frequencies, $M=$ the MTF of the display, and $m_{t}=$ the modulation threshold function of the observer. Thus, the Barten SQRI is calculated based on measurable properties of the visual displays and the contrast sensitivity function (CSF) of the person.

The Barten SQRI has not yet been applied to observers with low vision or SVDs. Low vision often causes decreased contrast sensitivity as well as reduced resolution, making it more difficult to see low-contrast images on SVD devices. In research with readers with normal vision, there is a sharp transition (critical scanning rate) from nearly perfect reading to poor reading due to changes in contrast [12]. When text has poor contrast and is blurred, reading is slowed only when the contrast drops below a critical contrast level, which is independent of character size. Studies with participants with normal vision indicate that readers without low vision are tolerant of changes in contrast and character size. In other words, people with normal vision do not show difficulty reading materials that have low contrast levels, such as LCD screens. However, people with low vision have less contrast and acuity reserves [13] and are not as tolerant to changes in contrast or low contrast levels as people without vision problems. In analyzing readers with normal vision, there tends to be an interaction between character size and contrast. Their CSFs indicate that sensitivities decline slowly for low frequencies (large characters) and decline more quickly for high frequencies (small characters). In other words, as characters become larger, contrast does not play as drastic a role as when characters become smaller. If the Barten SQRI accurately predicts how well individuals with low vision can read low-contrast SVD 
devices, it could be the first step in working toward universal design for SVD devices.

As a first step (proof of concept) toward evaluating whether the Barten SQRI formula predicts the ability of observers with low vision to access electronic devices with SVDs, this study tests whether the formula predicts the ability of individuals with low vision to recognize single characters with varying contrast levels on a CRT monitor.

\section{METHODS}

This study was approved by the institutional review board (IRB) at Emory University, Atlanta, Georgia. Participants were recruited from the Atlanta, Georgia, area. Inclusion criteria included age 18 and older with loss of central visual acuity measured with 5 percent low-contrast letters.

All visual measurements were performed using participants' best spectacle correction. Central visual field characteristics, including fovea and central scotoma characteristics, were measured with a scanning laser ophthalmoscope (SLO) (model 101, Rodenstock; Munich, Germany). The SLO obtains retinal images continuously with a nearly invisible infrared laser $(780 \mathrm{~nm})$ and scans graphics on the retina with a modulated red-light laser $(632 \mathrm{~nm})$ (Figure 1). The stimuli are thus observed by the subject and are seen directly on the subject's retina in real time [14].

Near visual acuity was tested with the Early Treatment of Diabetic Retinopathy Study chart at 100 and 5 percent contrast levels. Dark visual acuity was tested with the Smith-Kettlewell Institute Low Luminance (SKILL) chart. The SKILL chart assesses impairments in the ability to resolve letters in a low-contrast and lowluminance environment compared with a high-contrast and high-luminance environment (Figure 2). Dark visual acuity was measured because many SVD devices have poor contrast and are sometimes used in poorly lit home environments. Dark visual acuity is thus a better approximation than high-contrast visual acuity of the ability to resolve small print on SVDs.

Reading performance was measured with the SmithKettlewell Reading Test, which presents three lines of random letter and word phrases in decreasing font sizes (Figure 3). This test is sensitive to vision loss that affects visual skills for reading random words. That is, the test does not allow reading words based on the context of the sentence. This random word presentation method is more similar to what is presented by the Digit/Character Recognition Test

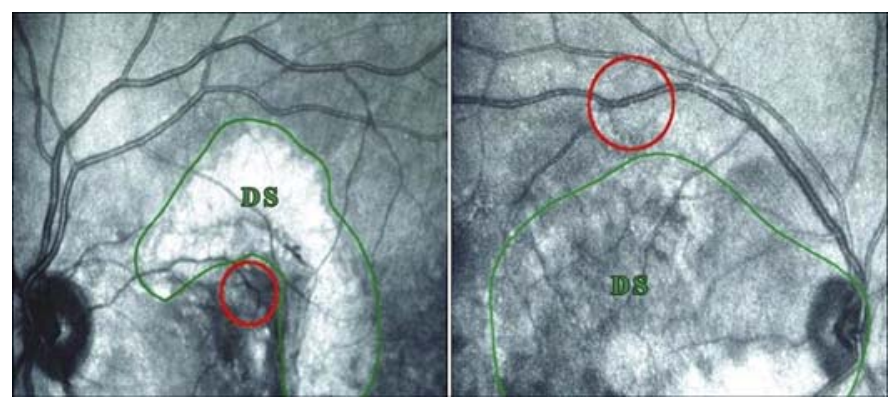

Figure 1.

Scanning laser ophthalmoscope images: green outline denotes dense scotoma (DS) while red circles indicate retinal fixation area or preferred retinal locus.



Figure 2.

Smith-Kettlewell Institute Low Luminance chart.

(see later) and by simple SVD devices (such as microwaves, blood-glucose monitors, clocks, and home thermostats), which tend to display numbers, words, or short phrases that have to be understood as stand-alone text rather than text on larger SVD devices (such as e-readers, smartphones, and small tablets) that benefit from continuous-print context. Each participant's maximum reading rate 


\section{so flash c box m sit $\mathrm{f}$ know tops $\mathrm{p}$ flash teach $r$ fear $d$ basket}

\author{
f sad $n$ free charm $r$ \\ do flaw sold $b$ clone \\ pulls s danger fan $n$
}

joy m old v note me
tear b light n stack
s land trees e walker

Figure 3.

Smith-Kettlewell Reading Test.

and critical print size were measured as variables that could potentially explain participants' ability to use SVDs. Though the present study involves only single characters on a display, the next phase of the project involves longer series of print. Reading rate was thus measured for the present study in preparation for the next phase.

Basic contrast sensitivity levels were determined with the Pelli-Robson Contrast Sensitivity Chart. CSF was found using a Visual Stimulus Generator (ViSaGe) system (Cambridge Research Systems; Rochester, United Kingdom), which is a graphics system that gamma corrects the contrast of the display using an OptiCAL photometer. Stimuli with accurate contrast were presented on a CRT display with the ViSaGe system. Square wave grating targets in four random orientations (horizontal, vertical, right diagonal, and left diagonal) at six increasing spatial frequencies $\left(0.5,1.0,2.0,4.0,8.0\right.$, and 16.0 cycles $\left./{ }^{\circ}\right)$ were presented while participants identified the orientation of each grating (Figure 4). Larger spatial frequencies (e.g., 16.0 cycles $/{ }^{\circ}$ ) are represented by thinner bars, and smaller spatial frequencies (e.g., 0.5 cycles $/{ }^{\circ}$ ) are represented with thicker bars. The MTF of the visual display was obtained from manufacturer specifications for the same range of spatial frequencies as used to find the CSF.

Dominant eye and preferred retinal locus (PRL) perception were determined by a binocular viewing test [15] using the ViSaGe system. Binocular pairs of targets were presented dichoptically and sequentially to the right or left eye using a monitor and LCD glasses system synched at 60 dichoptic pairs per second. Monocular PRL perception was determined based on whether the participant reported seeing just one of the images or a combined image from both eyes, i.e., a plus sign $(+)$, an " $X$ " $(\times)$, or a combination of the two (8-pointed star). Immediately after the dichoptic presentation, the single monocular target was presented to each eye to confirm that monocular perception alone was not inhibited (for example, that the monocular target was inside the scotoma of that eye). If the patient saw just one of the images binocularly and both of the monocularly presented images, it was determined that one eye was being suppressed and the other eye had the dominant PRL. If the combined image was seen, it was assumed that the brain was not suppressing one eye and thus neither PRL was dominant.

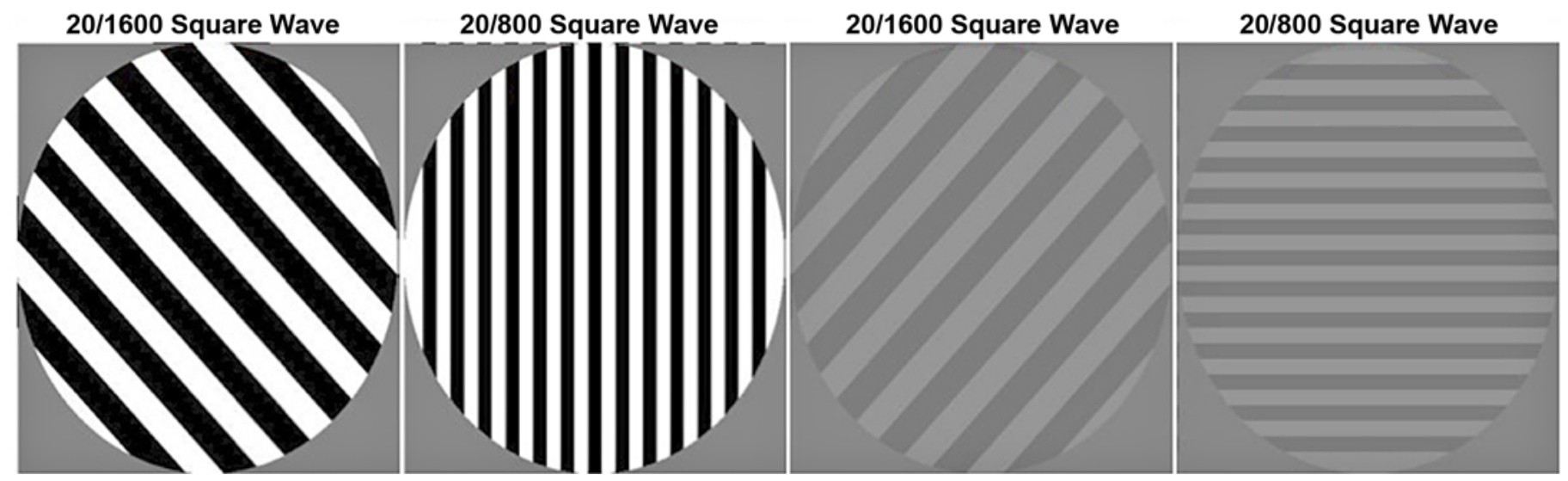

Figure 4.

Sample of square wave contrast sensitivity function stimuli as presented with Visual Stimulus Generator system. Throughout test, each combination of orientation, contrast, and frequency is presented. 
The Digit/Character Recognition Test measured the threshold contrast for each sized single digit. This test used the ViSaGe system to present stimuli that consisted of different size numbers at various contrast values. The single digit numbers (0-9) were presented in six decreasing heights $\left(8.00^{\circ}, 4.00^{\circ}, 2.00^{\circ}, 1.00^{\circ}, 0.50^{\circ}\right.$, and $\left.0.25^{\circ}\right)$. A two-up/one-down up-and-down transformed response rule adaptive staircase was used to determine the threshold contrast for number recognition by averaging eight reversals.

\section{RESULTS}

Thirty-three participants (18 males and 15 females) with macular disease affecting the fovea gave informed consent. Participants were 36 to 84 yr old (median age: 68 yr old). Participants had central scotomas in both, one, or neither eye with the frequency of 35, 39, and 26 percent, respectively. Participants had dominant perception in right, left, or both eye(s)/PRL(s) with the frequency of 42, 45, and 13 percent, respectively. Table 1 presents additional visual impairment measures. As would be expected, distance visual acuity scores were better for high-contrast charts (median: 0.78 logMAR or 20/120) than for low-contrast charts (median: 1.22 logMAR or 20/330). There was no significant difference $(p>0.05)$ between high-contrast distance visual acuity and high-contrast near visual acuity, but just as was found in distance visual acuity, the low-contrast, lowluminance near visual acuity (median: 2.00 logMAR or
20/2000) was significantly lower than high-contrast near visual acuity (median: 0.78 logMAR or 20/120). The critical print size for the participants was similarly reduced (median: 1.05 logMAR or 20/225). These reduced visual acuity scores with the reduced contrast sensitivity measures found for the participants (median: 1.20 logContrast) indicate that most of the participants were classified as participants with vision loss when looking at visual displays. In addition, most of the participants had central visual field loss (median: $12^{\circ}$ diameter macular scotoma), which would further reduce visual performance during visual tasks with visual displays.

Table 2 and Figures 5 to 9 present CSF, threshold contrast, and Barten SQRI results. Figure 5 shows five examples of the CSF found with the square wave CSF test. The participants either had the typical (compared with the CSFs of individuals with normal sight) peak sensitivity around 2 cycles $/{ }^{\circ}$, with decreased contrast sensitivity at both lower and higher spatial frequencies, or relatively flat contrast sensitivity up to 2 cycles $/{ }^{\circ}$ and then decreased contrast sensitivity at higher spatial frequencies. The Barten SQRI was calculated using the MTF of both the ViSaGe CRT and the participant, with the participant MTF found by the CSF test with square wave gratings. Figure 6 shows five examples of the contrast sensitivity required for threshold recognition of the Digit/Character Recognition Test. All participants had increasing contrast sensitivity with increasing height of characters, up to $2^{\circ}$ characters. With characters larger than $2^{\circ}$, some participants continued to increase

Table 1.

Visual impairment characteristics of participants.

\begin{tabular}{lcc}
\hline \multicolumn{1}{c}{ Visual Impairment and Functional Vision Test } & Median & Range (Min-Max) \\
\hline Visual Acuity (ETDRS Chart, logMAR) & $0.78(20 / 120)$ & $0.48-1.50^{*}(20 / 14-20 / 460)$ \\
$\quad$ 100\% Contrast & $1.22(20 / 330)$ & $0.54-2.00^{\dagger}(20 / 70-20 / 2000)$ \\
$\quad$ 5\% Contrast & $0.78(20 / 120)$ & $0.00-2.00(20 / 20-20 / 2000)$ \\
Near Visual Acuity (SKILL high-contrast chart, logMAR) & $2.00(20 / 2000)$ & $0.78-2.00(20 / 120-20 / 2000)$ \\
Dark Near Visual Acuity (SKILL low-contrast chart, logMAR) & $0-74$ \\
SKILL Score (no. of letters) & 44 & $0.30-1.60^{\ddagger}$ \\
Contrast Sensitivity (Pelli-Robson Contrast Sensitivity Chart, logContrast) & 1.20 & $0.0-20.0$ \\
Central Visual Fields (SLO, scotoma diameter [ $\left.\left.{ }^{\circ}\right]\right)$ & 12.0 & $0.0-110.3$ \\
Reading Rate Max (SKRead, words/min) & 28.6 & $0.10-1.30(20 / 25-20 / 400)$ \\
Critical Print Size (SKRead, logMAR) & $1.05(20 / 225)$ & \\
${ }^{*}$ Lower logMAR score indicates better visual acuity. & \\
${ }^{\dagger}$ Value of 2.00 indicates that no letters were read on chart. & \\
${ }^{\dagger}$ Higher logContrast score indicates better contrast sensitivity. & \\
ETDRS = Early Treatment of Diabetic Retinopathy Study, Max = maximum, Min = minimum, SKILL = Smith-Kettlewell Institute Low Luminance, SKRead = \\
Smith-Kettlewell Reading Test, SLO = scanning laser ophthalmoscope. \\
\hline \hline
\end{tabular}


in contrast sensitivity while others had relatively constant contrast sensitivities (plateaus on the graph) but with different peak values of contrast sensitivity. It is assumed that eventually even the participants with increasing contrast sensitivity with larger characters would have a relatively constant contrast sensitivity if even larger characters had been presented. But because this study is interested in SVD performance, character heights above $8^{\circ}$ are not relevant to the investigation.

Table 2.

Study outcome measures.

\begin{tabular}{lcc}
\hline \multicolumn{1}{c}{ Visual Stimulus Generator Research Tests } & Median & $\begin{array}{c}\text { Range } \\
\text { (Min-Max) }\end{array}$ \\
\hline Contrast Sensitivity Function (cycles ${ }^{\circ}$, logContrast) & & \\
0.5 & 1.55 & $0.35-1.95$ \\
1.0 & 1.65 & $0.15-2.05$ \\
2.0 & 1.55 & $0.20-1.95$ \\
4.0 & 1.10 & $0.00-2.00$ \\
8.0 & 0.30 & $0.00-1.55$ \\
16.0 & 0.00 & $0.00-0.80$ \\
Barten SQRI (just-noticeable difference) & 10.0 & $3.2-18.2$ \\
Digit/Number Recognition $\left(^{\circ}\right.$, logContrast) & & \\
0.25 & 0.00 & $0.00-0.80$ \\
0.50 & 0.15 & $0.00-1.25$ \\
1.00 & 0.50 & $0.00-1.60$ \\
2.00 & 1.05 & $0.10-1.70$ \\
4.00 & 1.15 & $0.20-1.70$ \\
8.00 & 1.15 & $0.50-1.75$ \\
\hline Max = maximum, Min = minimum, SQRI = square root integral. \\
\hline \hline
\end{tabular}

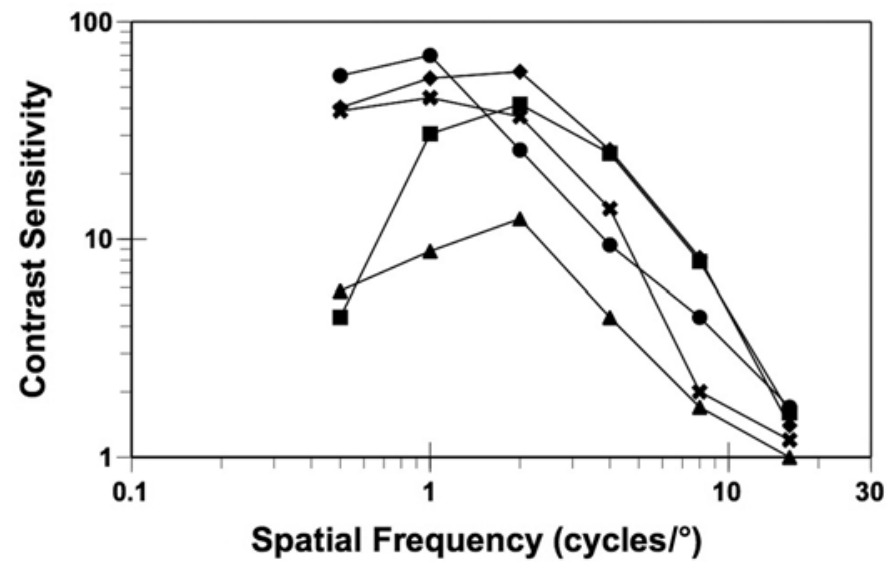

Figure 5.

Contrast sensitivity function (CSF), measured with square wave grating targets on Visual Stimulus Generator system, showing 5 typical sets of results out of 33 participants. CSF tends to be highest at medium spatial frequency while slightly lower at low spatial frequency and lowest at high spatial frequency.
Because all participants had relatively good contrast sensitivity with the $2^{\circ}$ characters and it is likely that most SVDs do not present larger than $2^{\circ}$ characters, Figure 7 shows a comparison of the relationship of $2^{\circ}$ digit recognition performance to the Barten SQRI. Also, threshold resolution (visual acuity) for all participants was sufficient such that performance in $2^{\circ}$ digit/character recognition was not limited by visual resolution. An analysis of variance shows that the $2^{\circ}$ digit threshold is significantly related $(p<0.01)$ to the Barten SQRI with a coefficient of determination $\left(r^{2}\right)$ of 0.78 . In comparison, a further analysis was done with the participants' best digit contrast sensitivity (at whatever size character was their individual best performance in threshold contrast sensitivity, which was always $\geq 2^{\circ}$ ) (Figure 8). It is possible, for example, that participants might use their handheld magnifiers (or some other magnifying aid) to increase the character heights above $2^{\circ}$ when using SVD devices. This analysis shows that the best contrast sensitivity (logContrast value) is significantly related $(p<0.01)$ to the Barten SQRI with a coefficient of determination $\left(r^{2}\right)$ of 0.80 . Finally, an analysis of variance was done to show the ability of the PelliRobson Contrast Sensitivity Chart to significantly predict performance on the Digit/Character Recognition Test. Figure 9 shows the significant relationship $(p<0.01)$ between the participant's best digit contrast sensitivity and the participant's Pelli-Robson Contrast Sensitivity Chart results with a coefficient of determination $\left(r^{2}\right)$ of 0.69 .

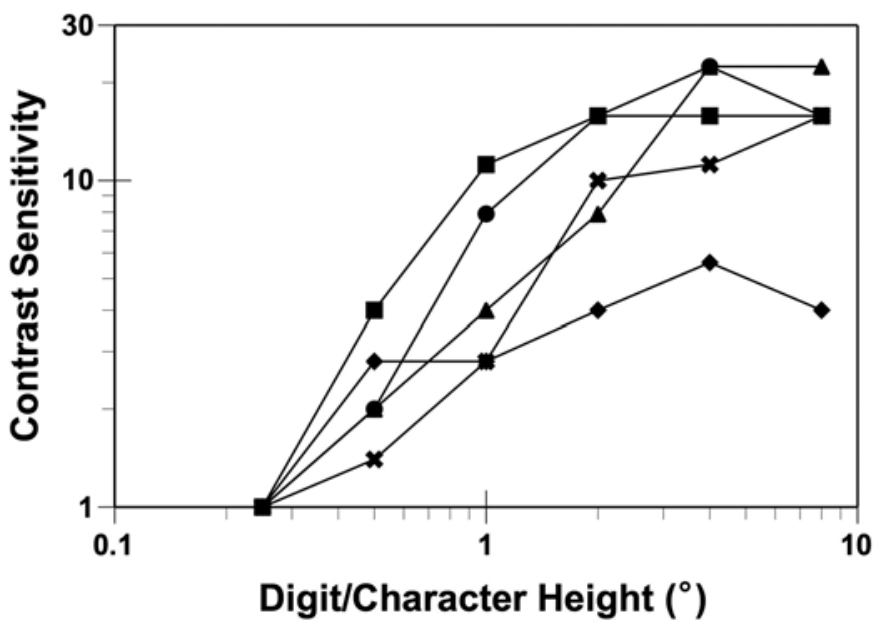

Figure 6.

Digit contrast threshold, measured with Digit/Character Recognition Test, showing 5 typical sets of results out of 33 participants. Threshold contrast sensitivity declines as digit height decreases. 


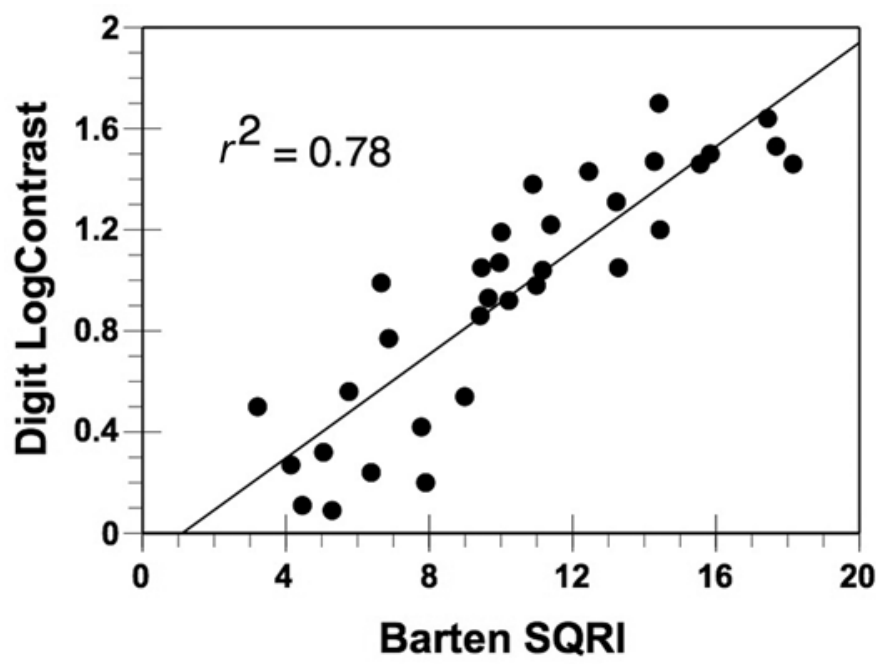

Figure 7.

Relationship of threshold logContrast for $2^{\circ}$ digit/character recognition to Barten square root integral (SQRI). $2^{\circ}$ digits/characters is sufficiently above threshold resolution (visual acuity) for all participants that performance in $2^{\circ}$ digit/character recognition was not limited by visual resolution.

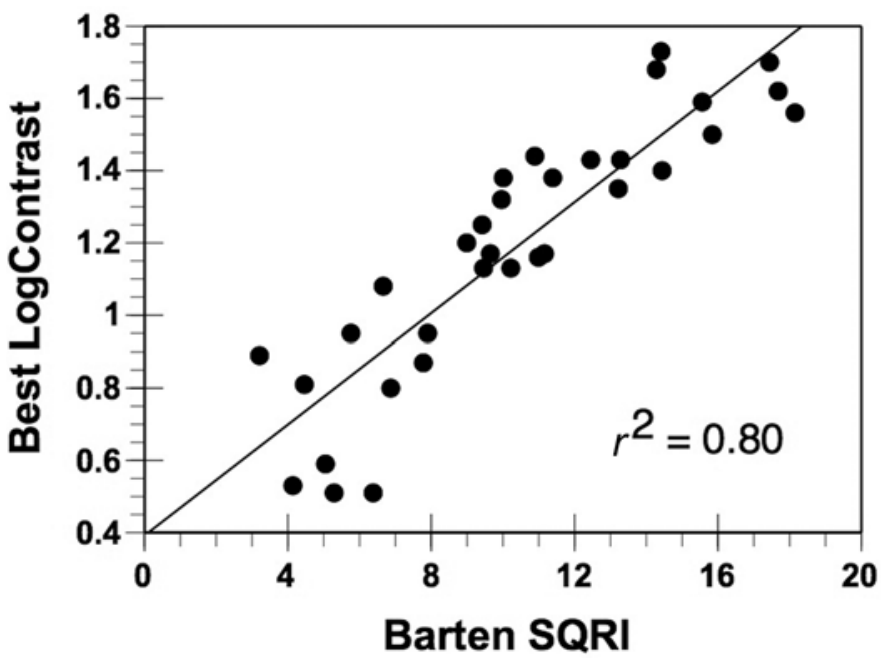

Figure 8.

Relationship of best threshold logContrast for digit/character recognition to Barten square root integral (SQRI). Relationship of most sensitive, or best, threshold contrast found for any digit/character size for each participant was found as function of Barten SQRI.

\section{DISCUSSION}

The best predictor of participants' ability to read threshold low-contrast digits on a CRT monitor was the Barten SQRI image metric. These findings in a sample

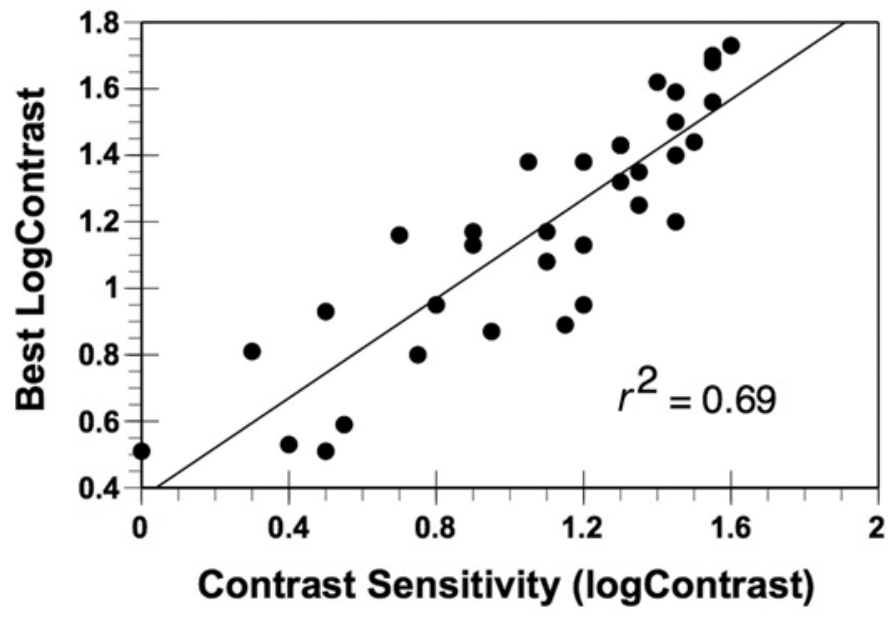

Figure 9.

Relationship of best threshold contrast for digit/character recognition to Pelli-Robson Contrast Sensitivity Chart. Best threshold contrast is found as described in Figure 8.

population with low vision are consistent with Barten's original findings among people with normal vision [11]. This study supports the hypothesis that the Barten SQRI shows potential to be used both to develop accessible SVDs on electronic devices and to help rehabilitation professionals match individual patients to the most effective devices for them.

While the Barten SQRI was an excellent fit for participants with low vision identifying single digits on a large monitor, using many SVD devices in real-life situations is much more complex than just reading or identifying characters on an SVD. The next step in testing the applicability of the Barten SQRI is to have participants with low vision use more textually complex SVD devices (such as smartphones and e-readers) to determine whether other variables interfere with usability and/or accessibility.

The practicality of using the Barten SQRI image metric in a rehabilitation service environment also must be determined. While the Barten SQRI was the best predictor of performance, the Pelli-Robson Contrast Sensitivity Chart was also a significant predictor. Given the current economic and political climate of the healthcare system in the United States, time and ease of testing must be considered when designing clinical assessments. For example, third-party payers reimburse low-vision occupational therapists one amount for an evaluation, regardless of the time it took to administer, score, and document the evaluation. A simpler, shorter test like the Pelli-Robson Contrast Sensitivity Chart may thus be more cost-effective 
than a test like the CSF that generally takes more time and needs more expensive equipment. Prior research has also shown that reading rate is more highly correlated with letter-contrast threshold than with grating-contrast threshold [16]. However, use of many SVD devices does not tend to require fast continuous print reading, and because the Pelli-Robson Contrast Sensitivity Chart does not incorporate the image characteristics of the display (which can vary much more than the high-resolution CRT used for this study), it may be necessary to further explore a combination of the simple contrast sensitivity test (like the Pelli-Robson or Rabin Contrast Sensitivity Charts) and the MTF of the display.

\section{CONCLUSIONS}

Measures of the person's contrast sensitivity and acuity alone may not be the best way to predict how well an individual with central vision loss will read text on a visual display in his or her daily life. If manufacturers and clinicians have a better understanding of how the contrast levels of different visual display devices affect users with low vision, then recommendations for designing visual display devices as well as vision rehabilitation programs could be tailored with different devices in mind.

\section{ACKNOWLEDGMENTS}

\author{
Author Contributions: \\ Study concept and design: M. Uslan, R. A. Schuchard. \\ Interpretation of data: K. A. Schoessow, L. M. Mauney, R. A. Schuchard. \\ Analysis of data: L. M. Mauney, R. A. Schuchard. \\ Drafting of manuscript: K. A. Schoessow, L. M. Mauney. \\ Critical revision of manuscript for important intellectual content: \\ M. Uslan, R. A. Schuchard. \\ Financial Disclosures: The authors have declared that no competing \\ interests exist.
}

Funding/Support: This material was based on work supported by the Department of Education and National Institute on Disability and Rehabilitation Research (grant H133G090026).

Additional Contributions: Dr. Schoessow is now with the Department of Occupational Therapy, Dominican University of California, San Rafael, California. Ms. Mauney is now with the Intel Corporation. Institutional Review: IRB consent was obtained from Emory University, the academic institution associated with the Atlanta Department of Veterans Affairs Medical Center where data was collected. All participants gave informed consent.

Participant Follow-Up: The authors do not plan to inform participants of the publication of this study.

\section{REFERENCES}

1. Roley SS, DeLany JV, Barrows CJ, Brownrigg S, Honaker D, Sava DI, Talley V, Voelkerding K, Amini DA, Smith TE, Toto P, King S, Lieberman D, Baum MC, Cohen ES, Cleveland PA, Youngstrom MJ; American Occupational Therapy Association Commission on Practice. Occupational therapy practice framework: domain \& process, 2nd edition. Am J Occup Ther. 2008;62(6):625-83. [PMID:19024744]

2. Uslan MM, Burton DM, Wilson TE, Taylor S, Chertow BS, Terry JE. Accessibility of home blood pressure monitors for blind and visually impaired people. J Diabetes Sci Technol. 2007;1(2):218-27. [PMID:19888410]

3. Cimarolli VR, Boerner K. Social support and well-being in adults who are visually impaired. J Vis Impair Blind. 2005; 99(9):521-34.

4. Cimarolli VR, Reinhardt JP, Horowitz A. Perceived overprotection: Support gone bad? J Gerontol B Psychol Sci Soc Sci. 2006;61(1):S18-23. [PMID:16399945] http://dx.doi.org/10.1093/geronb/61.1.S18

5. Reinhardt JP. Effects of positive and negative support received and provided on adaptation to chronic visual impairment. Appl Dev Sci. 2001;5(2):76-85. http://dx.doi.org/10.1207/S1532480XADS0502 3

6. Schoessow KA. Shifting from compensation to participation: A model for occupational therapy in low vision. $\mathrm{Br} \mathrm{J}$ Occup Ther. 2010;73(4):160-69. http://dx.doi.org/10.4276/030802210X12706313443947

7. Teitelman J, Copolillo A. Psychosocial issues in older adults' adjustment to vision loss: Findings from qualitative interviews and focus groups. Am J Occup Ther. 2005; 59(4):409-17. [PMID:16124207] http://dx.doi.org/10.5014/ajot.59.4.409

8. Kroemer KH, Grandjean E. Fitting the task to the human. 5th ed. London (United Kingdom): Taylor and Francis; 1997.

9. Shin-Tson W, Deng-Ke Y. Reflective liquid crystal displays. New York (NY): Wiley; 2001.

10. Vanderheiden GC, Vanderheiden KR. Guidelines for the design of consumer products to increase their accessibility to persons with disability or who are aging. Working draft 1.7 [Internet]. Madison (WI): Trace Research and Development Center, College of Engineering, University of WisconsinMadison; 1992. Available from: http://trace.wisc.edu/docs/ consumer product guidelines/toc.htm

11. Barten PG. Contrast sensitivity of the human eye and its effects on image quality. Bellingham (WA): SPIE Optical Engineering Press; 1999.

12. Legge GE, Pelli DG, Rubin GS, Schleske MM. Psychophysics of reading-I. Normal vision. Vision Res. 1985; 25(2):239-52. [PMID:4013091] http://dx.doi.org/10.1016/0042-6989(85)90117-8 
13. Whittaker SG, Lovie-Kitchin J. Visual requirements for reading. Optom Vis Sci. 1993;70(1):54-65. [PMID:8430009] http://dx.doi.org/10.1097/00006324-199301000-00010

14. Sunness JS, Schuchard RA, Shen N, Rubin GS, Dagnelie G, Haselwood D. Landmark-driven fundus perimetry using the scanning laster opthalmoscope. Invest Ophthalmol Vis Sci. 1995;36(9):1863-74. [PMID:7635660]

15. Schuchard RA, Hu S. Binocular preferred retinal loci: Relationship of visual factors to binocular perception. OSA Tech Dig Ser. 1995;1:332-35.

16. Rubin GS, Legge GE. Psychophysics of reading. VI-The role of contrast in low vision. Vision Res. 1989;29(1):79-91. [PMID:2788957]

http://dx.doi.org/10.1016/0042-6989(89)90175-2
Submitted for publication September 11, 2012. Accepted in revised form May 29, 2013.

This article and any supplementary material should be cited as follows:

Schoessow KA, Mauney LM, Uslan M, Schuchard RA. Evaluating Barten image metric for predicting character recognition in people with low vision. J Rehabil Res Dev. 2013;50(10):1343-52.

http://dx.doi.org/10.1682/JRRD.2012.09.0164

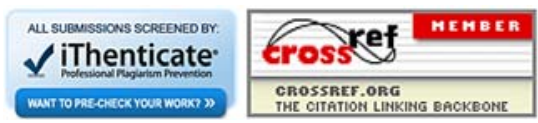


